\title{
Modelización del comportamiento del consumidor a favor de las energías renovables: Un estudio basado en las opiniones de los consumidores potenciales
}

\author{
Modeling behavior for renewable energies: A study based on the opinions of \\ potential consumers
}

\section{Modelização do comportamento do consumidor a favor das energias renováveis: Um estudo das opiniões de potenciais consumidores}

\author{
Diego Gómez Carmona \\ Universidad de Granada, España \\ gocadi@gmail.com \\ Francisco Muñoz-Leiva \\ Universidad de Granada, España \\ franml@ugr.es \\ Francisco J. Liébana-Cabanillas \\ Universidad de Granada, España \\ franlieb@ugr.es
}

\begin{abstract}
Resumen
En el presente trabajo se analiza el conocimiento que tienen los consumidores acerca de los diferentes tipos de energías renovables. Para modelizar el comportamiento del consumidor ante las energías renovables, la investigación basándose en la teoría de la acción razonada, relaciona de manera positiva el conocimiento previo sobre las energías renovables con la actitud. Tomando como punto de partida y variable exógena el comportamiento pro-ambiental, se demuestra la existencia de una relación fuerte entre esta variable y el conocimiento sobre las energías renovables. Por otra parte, entre esta preocupación por el medio, la actitud y la intención, se demuestra una relación menos intensa ya que la instalación de energías renovables supone un esfuerzo que frena al consumidor.
\end{abstract}

Palabras clave: adopción; comportamiento; energías renovables; modelización; Teoría de la Acción Razonada.

\begin{abstract}
This work analyses the consumer's knowledge of the different types of renewable energies. In order to model the consumers' behaviour regarding renewable energies, the research is based on the Theory of Reasoned Action, proposing a positive relationship between previous knowledge of the renewable energies and attitude. Considering the pro-environmental behaviour as a starting point and as an exogenous variable, this study demonstrates that there is a strong relationship between this variable and the knowledge of renewable energies. On the other hand, a less intense relationship has been demonstrated in the concern for the environment, attitude and intention to adopt, since the installation of renewable energies implies an additional effort that restrains the consumer.
\end{abstract}

Keywords: adoption; behaviour; renewable energies; modeling; Theory of Reasoned Action.

Dos Algarves: A Multidisciplinary e-Journal, $31-2017$.

ISBN 2182-5580 @ ESGHT - University of the Algarve, Portugal.

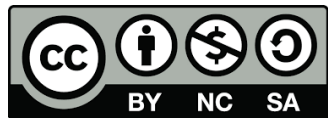

To cite this article: D. G. Carmona; F. Muñoz-Leiva e F. L. Liébana-Cabanillas (2017). Modelización del comportamento del consumidor a favor de las energias renovales: Un estúdio basado en las opiniones de los consumidores potenciales. Dos Algarves: A Multidisciplinary e-Journal, 31, 34-51. doi: 10.18089/DAMeJ.2017.31.3 


\section{Resumo}

Este trabalho analisa o conhecimento do consumidor sobre os diferentes tipos de energias renováveis. Para construir um modelo do comportamento dos consumidores em relação às energias renováveis, o estudo fundou-se na Theory of Reasoned Action, propondo uma relação positiva entre o conhecimento prévio das energias renováveis e a atitude. Considerando o comportamento pró-ambiental, como ponto de partida e como variável exógena, este estudo demonstra que existe uma forte relação entre essa variável e o conhecimento das energias renováveis. Por outro lado, uma relação menos intensa foi demonstrada na preocupação com o meio ambiente, atitude e intenção de adotar este tipo de energia, uma vez que a instalação de energias renováveis implica um esforço adicional que inibe o consumidor.

Palavras-chave: adoção; comportamento; energias renováveis; modelização; Theory of Reasoned Action.

\section{Introducción}

El modelo de desarrollo mundial está generando un cambio climático en todo el planeta, debido al consumo masivo de energías de origen fósil o la contaminación del aire y del agua entre otros motivos (Moreno \& López, 2008) además se ha demostrado que la acción humana es una de las principales causas del cambio climático, principalmente por el consumo de energía (García, 2012; Oreskes, 2004).

Por tanto, la situación actual demanda más que nunca un cambio en los modelos de gestión energéticos, en los que se racionalicen los recursos naturales y las prácticas sostenibles (Mitra, 2008); de manera que se reemplacen parte de las demandas energéticas tradicionales. Para esto, es necesario un cambio no solo en valores y actitudes sino también en los comportamientos individuales (Gardner \& Stern, 2008). Para entender de qué forma el consumidor y su comportamiento de consumo influye en el mercado, se debe partir del conocimiento previo de variables ambientales y el impacto de las actividades de marketing, así como su relación con decisiones de marketing empresarial (Chamorro, Rubio \& Miranda, 2009). Además, el estudio sobre los consumidores y los mercados verdes o ecológicos se debe complementar cada vez más con el análisis de otras variables, tal y como Castañeda (2014) recomienda; en especial aquellas relacionadas con el consumidor bajo una perspectiva psicosocial, teniendo en cuenta variables como actitudes, motivaciones o preocupación por el medio ambiente, entre otras. Este trabajo, modeliza el comportamiento del consumidor bajo esta perspectiva psicosocial basándonos en el hecho de que la instalación de energías de la biomasa, la energía solar térmica y la energía solar fotovoltaica depende fuertemente de una decisión personal.

Para tal objetivo se presentan las hipótesis de investigación que aparecen descritas en el siguiente epígrafe. En el epígrafe tercero se concretan los aspectos metodológicos de la investigación. A continuación, en la sección de resultados se exponen los principales hallazgos derivados del análisis de datos. Por último, a partir del quinto epígrafe se extraen las principales conclusiones y recomendaciones.

\section{Revisión bibliográfica}

\subsection{Modelización del comportamiento a favor de las energías renovables}

Las investigaciones sobre energías renovables, han abordado tanto la influencia que tienen los factores culturales y sociales, así como los psicológicos en la demanda de este tipo de instalaciones (Clark, Kotchen \& Moore, 2003; Vringer, Aalbers, \& Blok, 2007). Las variables 
clave medidas en nuestro modelo son el comportamiento pro-ambiental o preocupación ambiental, el conocimiento previo sobre las energías renovables, la actitud hacia las instalaciones alternativas a las tradicionales y la intención de compra de las distintas fuentes de energías renovables.

En el presente estudio, tanto la preocupación por el medio, puesta de manifiesto a través del activismo y el ahorro de energía, así como las creencias personales sobre el conocimiento de las energías renovables consideradas, son los principales determinantes de la actitud hacia el consumo de estas fuentes de energía (Diamantopoulos, Schlegelmilch, Sinkovics \& Bohlen, 2003; Camarena Urbano, 2013). Estas variables serán abordadas a continuación.

\subsubsection{La preocupación por el medioambiente}

El comportamiento pro-ambiental, engloba comportamientos relacionados con la protección del medio ambiente y que suponen poco esfuerzo (Cortés-Peña, 2016).

Los factores antecedentes de la preocupación ambiental han proporcionado gran cantidad de literatura desde sus inicios, que puede ser diferenciada por etapas.

En una primera etapa, se puede distinguir a Maloney y Ward (1973) que pretendían conocer los motivos individuales que llevan a un comportamiento responsable con el medio ambiente. Autores como Dunlap y Van Liere (1978) indicaron que en los países occidentales se estaba produciendo un cambio en la visión del mundo pasando a lo que denominaron el "Nuevo Paradigma Ambiental", que se preocupaba por aspectos de estética ambiental y ahorro de energía. Otros investigadores, como Slovic (1987) o Gould et al. (1988) consideraron que la preocupación medioambiental, procedía de los riesgos que podrían traer los problemas ambientales.

En la segunda etapa, investigadores como Jones y Dunlap (1992), Guagnano, Stern y Dietz (1995), Stern, Dietz, Gregory y Guagnano (1995), Karp (1996), Séguin, Pelletier y Hunsley (1999) y Blake (1999) planteaban que los factores demográficos como la edad, los ingresos o la educación así como valores morales, eran los que llevaban a los individuos a estar preocupados por el medio.

En la actualidad, la mayor preocupación se centra en los problemas particulares de los distintos países ya que los problemas ambientales se han agravado y se empieza a considerar el sobrecalentamiento global (Alonso, 2013). Debido a esto, algunos de los factores a lo que más importancia se le está dando son al ahorro de energía y al activismo como demuestran los estudios de Kelly, McDonald y Winnifred (2008), Fielding, Terry, Masser y Hogg (2008), Dono, Webb, y Richardson (2010), Senbel, Douglas y Blair (2014) y García et al. (2016). En el trabajo se ha utilizado para explicar el comportamiento pro-ambiental, tanto el activismo proambiental como el ahorro de energía.

- El activismo ha sido objeto de estudio por muchas de las investigaciones de la psicología ambiental. En particular, Pelletier, Tuson, Green-Demers, Noels, \& Beaton (1998: 632) lo define como: "la realización de comportamientos específicos". Esta definición engloba distintos comportamientos, como pueden ser la pertenencia a un grupo ambiental participar en acciones políticas a favor del medio ambiente, realizar intencionadamente un comportamiento comprometido con el medio o involucrarse en conductas de protección del medio ambiente (Imhoff, Gariglio, Ponce, Díaz \& Pilatti, 2014). 
- El ahorro de energía está relacionado directa e indirectamente con el comportamiento de consumo de energía de los distintos individuos que habitan en un hogar. Este ahorro es utilizado por los investigadores como un indicador del comportamiento pro-ambiental (Cortés-Peña, 2016).

Los comportamientos que tienen las personas en sus hábitos diarios están relacionados con acciones específicas y coherentes como pueden ser, el ahorro de energía o la compra de productos respetuosos con el medio ambiente (Izagirre-Olaizola, Fernández-Sainz \& VicenteMolina, 2013). Esta agrupación de comportamientos ambientales responsables se han observado en diferentes países y pueden facilitar una transición hacia estilos de vida que mejoren el medio ambiente (Whitmarsh \& O’Neill, 2010).

Este comportamiento pro-ambiental resulta ser un factor determinante, tanto directo como indirecto de los comportamientos ambientales específicos, como puede ser la adquisición de energías renovables (Bamberg, 2003).

\subsubsection{El conocimiento previo}

El conocimiento previo tiene un impacto significativo en la evaluación de las posibles alternativas, así como en las decisiones de compra Carrero, Valor y Redondo (2015).

Las personas pueden adquirir conocimientos, mediante sus propias experiencias, a través de terceros o mediante estímulos visuales, verbales y sensoriales, tales como anuncios, textos periodísticos, revistas o programas de televisión (Huang, Gursoy \& Honggang, 2014). Siguiendo a Carrero et al. (2015) se asume que el consumidor utiliza primero el conocimiento que tiene a la hora de realizar una compra ya que este procesamiento de la información es más fácil y más eficiente.

Nelson, Bishop y Gruen (2014) indica que el conocimiento sobre productos de eficiencia energética, puede contribuir de una manera fuerte, a la formación de actitudes hacia este tipo de productos.

El conocimiento previo, ha sido utilizado en anteriores investigaciones por investigadores como Miguens, González y Vázquez, (2015). Concluyendo que mayores niveles de conocimiento no se relacionan con la actitud, sino con la intención. Bien es cierto que esta investigación indagaba sobre el comportamiento ecológico en general. En el presente trabajo mediremos el conocimiento subjetivo sobre las energías renovables de los individuos encuestados.

\subsection{El papel de la actitud y la intención de adopción}

\subsubsection{La actitud hacia las energías renovables}

En las investigaciones que encontramos sobre actitudes hacia las energías renovables, indican un apoyo generalizado a estas fuentes de energía (Sengers, Raven \& Van, 2010). Los trabajos de Sardianou y Genoudi, (2013), indicaban que eran los factores personales y contextuales los que explican las actitudes del público hacia este tipo de fuentes de energía.

Stigka, Paravantis y Mihalakakou (2014) identificaron actitudes de apoyo de los consumidores a la hora de tomar las decisiones sobre el tipo de energía a consumir, indicando que estas decisiones estaban motivadas por la protección al medio ambiente. 


\subsubsection{La predisposición a adoptar energías renovables}

La importancia del estudio de las intenciones de compra de energía renovable está relacionada por la cercanía entre esta intención y el comportamiento de compra futuro. Estas relaciones se describen en la teoría de la acción razonada, TRA (Ajzen \& Fishbein, 1980) y en la teoría del comportamiento planeado - TPB (Ajzen, 1991).

La teoría TPB plantea una fuerte relación entre actitud e intención, apoyada en muchos campos de investigación (Chan, Wu \& Hung, 2010), pero la relación que se da entre la intención y el comportamiento tiene menos apoyo en el ámbito medioambiental. A esta diferencia entre las intenciones de compra y el comportamiento real se le ha llamado como "brecha de acción" por los investigadores Claudy, Peterson y O’Driscoll (2013).

\subsection{Planteamiento de las hipótesis de investigación}

\subsubsection{Efectos del comportamiento pro-ambiental y el conocimiento previo}

En este trabajo, la preocupación ambiental que se pone de manifiesto al realizar una serie de comportamientos responsables con el medio ambiente, ha sido utilizada como un antecedente del conocimiento previo sobre las energías renovables, de la actitud y de la intención de compra de las mismas. Los consumidores que en su vida diaria llevan a cabo un comportamiento pro-ambiental, dicen preferir productos respetuosos con el medio ambiente (Stigka et al., 2014).

Cuando un asunto involucra valores personales como el futuro de los hijos o la protección del medio, aumenta la búsqueda de información (Zaichkowsky, 1985). Esta búsqueda puede dar lugar a mayores niveles de conocimiento de los consumidores acerca de las posibles soluciones alternativas de los problemas ambientales, como son las energías renovables. En consecuencia, debemos esperar que aquellas personas que están más preocupadas por el medio ambiente y así lo demuestren mediante sus comportamientos proambientales, posean un mayor conocimiento previo sobre las energías renovables estudiadas (Zsóka, Szerényi, Széchy \& Kocsis, 2012).

Derivado de los argumentos anteriores planteamos la siguiente hipótesis de investigación.

H1: Cuanto más se ejerza un comportamiento pro-ambiental mayor será el conocimiento de las energías renovables.

\subsubsection{Efectos del comportamiento pro-ambiental}

Los problemas ambientales como el calentamiento global o el cambio climático, harán surgir el interés de las energías renovables (Li, Jenkins-Smith, Silva, Berrens \& Herron, 2009).

La discrepancia entre el comportamiento pro-ambiental y las intenciones de compra de las energías renovables, se debe a variables que moderan esta influencia (Bamberg, 2003). Ha y Janda (2012) indicaban que las actitudes a favor de la conservación de energía son más probables que se conviertan en una acción, si dicha acción tiene un coste pequeño en referencia a tiempo, dinero o sacrificio.

En la aplicación de la teoría TRA de Ajzen y Fishbein (1980), para el caso de las energías renovables, se indica que la actitud hacia las energías renovables, media entre el efecto del 
comportamiento pro-ambiental y la intención de compra (Lin \& Syrgabayeva, 2016). Hansla, Gamble, Juliusson y Gärling (2008) descubrieron que había influencia directa del comportamiento pro-ambiental sobre la intención y una influencia indirecta mediante las actitudes sobre la intención de los consumidores al comprar energías alternativas. La TRA indica que la conducta de un individuo está influenciada por su intención.

H2: Cuanto más se ejerza un comportamiento pro-ambiental, mayor será la actitud hacia las energías renovables.

H3: Cuanto más se ejerza un comportamiento pro-ambiental, mayor será la intención de compra de energías renovables.

\subsubsection{Efectos del conocimiento previo y la actitud}

Egea y Frutos (2013) defienden que la relación positiva entre el conocimiento y las actitudes indican que hay más factores que afectan a la actitud hacia las energías renovables. Estos factores, son los denominados "factores situacionales" que incluyen tanto las limitaciones económicas, presiones sociales y las oportunidades para elegir distintas formas de suministro de energía (Hines, Hungerford \& Tomera, 1987). Los individuos que llevan a cabo un comportamiento pro-ambiental pueden tener una actitud más positiva hacia estas energías verdes, ya que son conscientes de las ventajas de instalar sistemas de ahorro, basados en renovables (Lin \& Syrgabayeva, 2016).

Pagiaslis y Krontalis (2014) en sus trabajos indicaban que a medida que los consumidores tienen más conocimiento de las ventajas relativas de las energías alternativas, están dispuestos a pagar más por estas fuentes de energía.

En base a lo planteado por la literatura sugerimos la siguiente hipótesis de investigación.

H4: Un mayor conocimiento previo sobre las energías renovables tiene una influencia positiva sobre la actitud hacia estas energías.

\subsubsection{Efecto de la actitud del individuo}

Según la teoría TRA de Ajzen y Fishbein, (1980), se plantea la existencia de una relación positiva y fuerte entre la actitud del individuo y la intención de realizar dicho comportamiento. Si una persona realiza un juicio evaluativo favorable, sus actitudes darán lugar a intenciones de realizar un determinado comportamiento (Wolske, Stern \& Dietz, 2017). Según la TRA la intención es el principal predictor de la conducta de compra, sin embargo, esta relación se ha planteado en productos o servicios en los que no existían impedimentos que puedan frenar la intención de realizar el comportamiento.

H5: Una actitud favorable hacia las energías renovables tiene un efecto positivo sobre la intención de compra hacia los distintos tipos de instalaciones basadas en energías renovables. 
Figura 1. Modelo propuesto

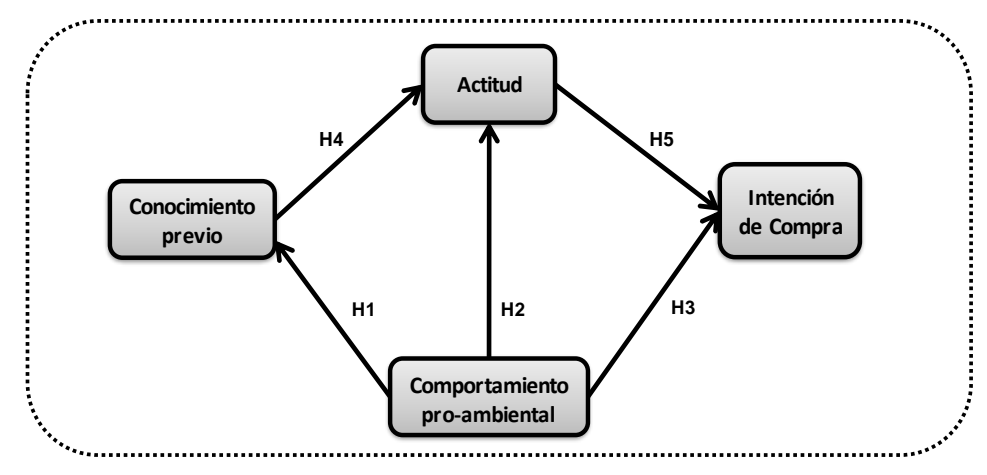

\section{Metología de investigación}

A continuación presentamos los principales aspectos de la metodología, que hacen referencia al cuestionario utilizado, confeccionado tras una revisión de la literatura científica sobre el tema bajo estudio y sometido a una revisión crítica por parte de 3 expertos en medio ambiente, se programó con la herramienta de formularios on-line, de google drive (ver Anexo 1). Incluye escalas de medida tipo Likert con cinco posibles respuestas, siendo 1 (menor valor) y 5 (mayor valor) las posiciones extremas. En la introducción del cuestionario se informa que las respuestas serán anónimas, se asegura que no hay respuestas correctas o incorrectas y que deben responder con la mayor sinceridad posible. Esta indicación permite reducir los sesgos comunes del método, especialmente en la etapa de recogida de datos. En particular, esta aclaración puede mitigar cualquier tipo de inquietud en la evaluación de los participantes y hacer que sean menos propensos a dar respuestas más deseables, indulgentes, aquiescentes y consistentes con la forma en que piensan que la investigación quiere que respondan (Podsakoff, MacKenzie, Lee \& Podsakoff, 2003).

\subsection{Características de la muestra y recogida de datos}

La población objeto de estudio quedó definida como los propietarios de viviendas residenciales en España, ya que son los que tienen independencia para tomar decisiones sobre el sistema energético a utilizar en el hogar. En concreto podemos decir que la propiedad de la vivienda, influye en la inversión en sistemas de ahorro energético (Frederiks, Stenner \& Hobman, 2015). La investigación se centró en inmuebles unifamiliares (adosados o pareadas) y en viviendas integradas en edificios (bloques de pisos). El método de recogida de datos estuvo basado en un muestreo por conveniencia, utilizando la encuesta personal online con cuestionario estructurado y precodificado. La selección de la muestra se realizó compartiendo la publicación en Facebook, Twitter y mediante correo electrónico. La participación en el estudio fue voluntaria. El trabajo de campo se realizó durante los meses de abril a julio de 2014 y se garantizó la confidencialidad y privacidad de los entrevistados.

La base de datos inicial, estaba compuesta de 253 encuestas (ver Tabla 1), tras la aplicación de depuración de la muestra utilizando sendos criterios de tiempo de respuesta y propiedad de la vivienda, llevaron a la eliminación de respuestas con una duración inferior a 5 minutos (speeders) y de individuos que no tenían la vivienda en propiedad, obteniendo un total de 218 cuestionarios válidos. 
Tabla 1. Ficha técnica del estudio

\begin{tabular}{|l|l|}
\hline Trabajo de campo & De abril a julio de 2014. \\
\hline Población & Usuarios potenciales de energías renovables. \\
\hline Tamaño de la población & 18.217 .300 hogares en España en 2013. \\
\hline Tipo de muestreo & $\begin{array}{l}\text { Por conveniencia. Autoselección mediante invitación por red } \\
\text { social Facebook, Twitter y mediante correo electrónico. }\end{array}$ \\
\hline Tipo de encuesta & A través de cuestionario web. \\
\hline Duración media de la encuesta & 11 minutos, 20 segundos. \\
\hline Tamaño de la muestra & 218 cuestionarios válidos de 253 iniciados. \\
\hline Error muestral & $6,64 \%$, suponiendo $\mathrm{p}=\mathrm{q}=0,5$ y nivel de confianza del $95 \%$ \\
\hline
\end{tabular}

* Bajo los supuestos de muestreo aleatorio simple

Por último, partiendo de los principios del muestreo aleatorio simple (m.a.s.), para un nivel de confianza del $95 \%$ y en la hipótesis más desfavorable de máxima indeterminación $(\mathrm{P}=\mathrm{Q}=0,5)$, el margen de error de los datos para la estimación de una proporción fue de $6,64 \%$.

\subsection{Escalas utilizadas para medir las variables del estudio}

El cuestionario web utilizado se encuentra disponible en Anexo 1. Con respecto a las escalas de medida empleadas, la escala de comportamiento ecológico (ECE) está basada en la escala de comportamiento pro-ambiental de Karp (1996). El ahorro de energía, así como el activismo, han sido utilizadas por Senbel et al. (2014) en un estudio reciente realizado por la universidad británica de Columbia.

En este trabajo, la escala ECE ha sido adaptada al problema de la energía utilizando 14 ítems en una escala tipo Likert (donde 1 es nunca y 5 es siempre) referentes a dos de las dimensiones, las cuales han sido validadas (mediante un análisis factorial exploratorio). La bidimensionalidad de la escala quedó demostrada con un análisis factorial exploratorio que arrojaba una varianza explicada del 65,73\%.

El análisis de fiabilidad a partir de los indicadores de consistencia interna (alpha de Cronbach o Fiabilidad Compuesta - SCR) obtuvieron valores mayores al utilizado como referencia $(0,70)$ en la literatura (Venkatesh, Morris, Davis \& Davis, 2003; Ranganathan \& Ganapathy, 2002). En concreto el estadístico alpha de Cronbach mostró un valor del 91,8\%. Niveles altos de fiabilidad $(\geq 0,90)$ permiten que en el análisis de datos puedan obtenerse variables resumen o suma como buenos indicadores de los constructos que recogen la variabilidad de los datos. Esta decisión presidirá la formación de niveles generales de las dimensiones de esta escala de medida (Rifon, LaRose \& Choi, 2005). Así los seis primeros ítem (ver Anexo 1) se promediaron en una variable resumen llamada "activismo". Por su parte los ocho ítems restantes (ver Anexo 1) referentes al ahorro de energía también fueron promediados y recogidos por la variable "ahorro energético".

La escala de conocimiento previo subjetivo utilizada en el trabajo, responde a una Likert formada por 5 ítems (que oscila desde 1 que es totalmente en desacuerdo hasta 5 totalmente de acuerdo) fue adaptada de la escala utilizada por Rijnsoever y Farla (2014). En estos análisis, se puso de manifiesto la unidimensionalidad de la escala que llega a explicar un 68,59\% de la varianza. A través del valor alpha se demostró la consistencia interna de la escala ya que el resultado de este análisis es del $88,5 \%$. 
La actitud fue medida en este trabajo, a partir de una escala clásica de un solo ítem tipo Likert de cinco puntos (donde 1 es nada favorable y 5 es muy favorable) adaptada de Bruner (2009).

La intención fue medida con una escala tipo Likert de cuatro ítems (donde 1 es totalmente en desacuerdo y 5 es totalmente de acuerdo). Los ítems de esta escala hacen referencia a la intención de compra futura de cada uno de los tres tipos de energías renovables que se contempla en el estudio y el último considera la intención de compra futura de energía renovable en general. Fue adaptada de la escala de Jamieson (1989). La unidimensionalidad de la intención quedó demostrada mediante un análisis factorial cuya varianza explicada es del $62,53 \%$ y la consistencia interna de la escala analizada con el estadístico alpha de Cronbach fue de un $80 \%$.

Todas las escalas de medida utilizadas en el trabajo son consistentes internamente, ya que su alpha de Cronbach es superior a 0,70, por tanto, podemos decir que son generalmente aceptadas y poseen unos indicadores de fiabilidad adecuados (Churchill, 1979).

Para contrastar las hipótesis planteadas, se ha utilizado un modelo de ecuaciones estructurales, en el que el comportamiento pro-ambiental fue considerado como una variable exógena y tanto el conocimiento previo, como la actitud y la intención de compra de energías renovables son consideradas como variables endógenas. Para analizar los datos se utilizó el software AMOS 18.

El análisis univariante previo mostró que los ratios críticos referentes a la simetría seguían una distribución normal, pero los que se refieren a curtosis no se distribuían de la misma manera que la distribución normal, por lo que se concluyó que existían desviaciones respecto a la normalidad univariante. El estadístico de curtosis multivariante de Mardia, que considera la distribución conjunta de la muestra es de 2,86 con un valor crítico de 1,16 que se encuentra dentro de la región de aceptación $( \pm 1,96)$, por tanto, se encontró evidencia empírica para considerar que la muestra sigue una distribución normal multivariante. Teniendo en cuenta los datos y con un tamaño muestral superior a 200 se utilizó el método de máxima verosimilitud para la estimación del modelo.

\section{Análise de datos}

\subsection{Modelización de la potencial adopción de energías renovables}

4.1.1. Fiabilidad y validez de las escalas de medida

A continuación aparecen los principales resultados de la fiabilidad y validez de las escalas de medida empleadas en la modelización del comportamiento de adopción de energías renovables (ver Tabla 2).

En primer lugar, la fiabilidad compuesta de la escala de comportamiento pro-ambiental es de casi el $66 \%$, aunque se encuentra cerca del límite establecido del $70 \%$ no llega a superarlo. La varianza extraída es de 0,5 por lo que se podría decir que el comportamiento pro-ambiental comparte el $50 \%$ de la varianza con sus indicadores, superando los límites de corte recomendados por la literatura científica.

La fiabilidad compuesta del conocimiento previo nos indica que esta escala explica por término medio un $89 \%$ de sus indicadores, superando el límite de corte recomendado por la literatura. La varianza extraída es de 0,61 alcanzando el valor establecido como referencia por la literatura 0,50 , considerándose una escala con adecuadas propiedades sicométricas. 
Tabla 2. Fiabilidad compuesta y varianza extraída

\begin{tabular}{|c|c|c|c|c|c|c|}
\hline $\begin{array}{c}\text { Variables } \\
\text { observadas }\end{array}$ & $\begin{array}{l}\text { Coeficiente } \\
\text { estandarizado }\end{array}$ & $\mathrm{R}^{2}$ & $\mathrm{CE}^{2}$ & $1-R^{2}$ & $\begin{array}{l}\text { Fiabilidad } \\
\text { compuesta }\end{array}$ & $\begin{array}{l}\text { Varianza } \\
\text { extraída }\end{array}$ \\
\hline ahorro & 0,776 & 0,60 & 0,60 & 0,40 & \multirow{2}{*}{0,66} & \multirow{2}{*}{0,50} \\
\hline activismo & 0,624 & 0,39 & 0,39 & 0,61 & & \\
\hline $\mathrm{CP} 1$ & 0,796 & 0,63 & 0,63 & 0,37 & \multirow{5}{*}{0,89} & \multirow{5}{*}{0,61} \\
\hline $\mathrm{CP} 2$ & 0,755 & 0,57 & 0,57 & 0,43 & & \\
\hline $\mathrm{CP}_{3}$ & 0,773 & 0,60 & 0,60 & 0,40 & & \\
\hline $\mathrm{CP}_{4}$ & 0,79 & 0,62 & 0,62 & 0,38 & & \\
\hline $\mathrm{CP}_{5}$ & 0,784 & 0,61 & 0,61 & 0,39 & & \\
\hline Int1 & 0,729 & 0,53 & 0,53 & 0,47 & \multirow{4}{*}{0,80} & \multirow{4}{*}{0,50} \\
\hline Int2 & 0,727 & 0,53 & 0,53 & 0,47 & & \\
\hline Int3 & 0,74 & 0,55 & 0,55 & 0,45 & & \\
\hline Int4 & 0,628 & 0,39 & 0,39 & 0,61 & & \\
\hline
\end{tabular}

La intención de adopción obtiene una fiabilidad compuesta del 80\%, siendo superior al 0,70 que se establece como límite de corte recomendado en la literatura. La varianza extraída de la intención es de 0,5 con lo que podemos decir que también cumple el corte recomendado por la literatura.

Las escalas globalmente consideradas podrían considerarse buenas ya que, aunque uno de los indicadores del comportamiento pro-ambiental es inferior al límite establecido por la literatura, los indicadores de las escalas restantes son buenos.

\subsubsection{Modelo de comportamiento a favor de las energías renovables}

4.1.2.1 Ajustes globales del modelo: A continuación aparecen los valores de los indicadores de bondad del ajuste global del modelo (ver Tabla 3).

Tabla 3. Indicadores de ajuste global

\begin{tabular}{|c|c|c|c|c|c|}
\hline Chi-cuadrado & p-valor & GFI & AGFI & CFI & RMSEA \\
\hline 69,375 & 0,36 & 0,950 & 0,922 & 0,983 & 0,042 \\
\hline
\end{tabular}

Los ajustes alternativos (a la Chi-cuadrado) calculados, como el GFI que representa el grado de ajuste global cumple con los requisitos marcados por la literatura $(>0,90)$. El AGFI, que considera el ajuste global del modelo teniendo en cuenta el número de parámetros se considera igualmente adecuado (>0,90). El CFI índice de ajuste que compara el modelo estimado con el modelo nulo, también alcanza un valor adecuado (próximo a 1). El RMSEA, indicador que tiene en cuenta los residuos que genera el modelo calculado es inferior a $(0,05)$ valor recomendado por la literatura.

Todos los indicadores, excepto la Chi-cuadrado, muestran que el modelo reproduce razonablemente bien la matriz de varianzas-covarianzas de la población. 
4.1.2.2. Significación de los coeficientes estimados: Para contrastar las hipótesis recogidas en la literatura analizaremos los coeficientes estandarizados.

Figura 2. Modelo de comportamiento hacia las energías renovables extraído. Coeficientes estandardizados

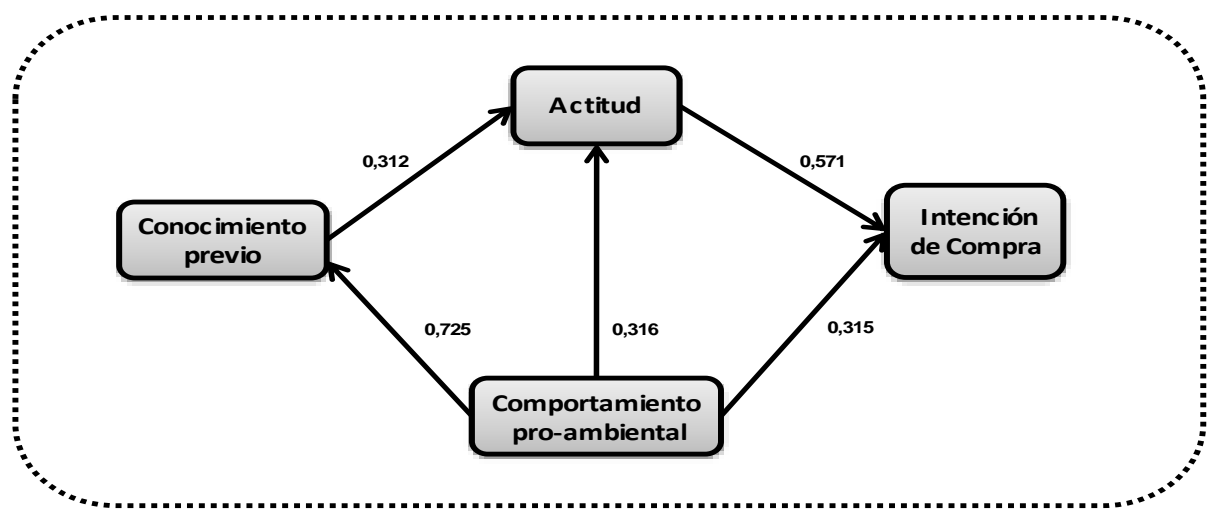

Como se puede observar (ver Figura 2), las relaciones recogidas en el modelo propuesto son positivas y significativamente distintas de cero confirmando todas las hipótesis de investigación. Además, el modelo explica un porcentaje importante (61\%) de la variabilidad de la intención de compra de energías renovables.

En primer lugar, existe evidencia empírica para apoyar la primera hipótesis de investigación ( $\mathrm{H} 1$ ) confirmándose una relación fuerte entre el comportamiento pro-ambiental y el conocimiento previo de las energías renovables, con un coeficiente estandarizado significativo superior a $0,5(B=0,725$; sign. $=0,00)$. Por tanto, se descubre que a mayor preocupación o comportamiento pro-ambiental se tiende a ser más conocedor de las energías renovables.

La hipótesis $\mathrm{H} 2$, que sostiene una relación entre comportamiento pro-ambiental y la actitud hacia las energías renovables, queda confirmada con una relación algo más moderada entre dichas variables $(B=0,316$; sign. $=0,0016)$, al estar el coeficiente estandarizado entre 0,1 y 0,5 . De esta forma se descubre que cuanto mayor es el comportamiento pro-ambiental, mayor será la actitud hacia las energías renovables consideradas (biomasa, solar y fotovoltaica).

La relación que plantea la $\mathrm{H}_{3}$, entre comportamiento pro-ambiental e intención de compra, es moderada y significativa al igual que en el caso anterior $(\theta=0,315$; sign. $=0,000)$. De esta forma se da a conocer que cuanto mayor es el comportamiento pro-ambiental, mayor será la intención de compra de energías renovables.

La $\mathrm{H}_{4}$ que establece la relación entre el conocimiento previo y la actitud se confirma, mostrando una influencia en la relación de dimensión similar a las dos relaciones anteriores $(b=0,312$; sign. $=0,008)$. De esta manera se descubre que cuanto mayor es el conocimiento previo sobre las energías renovables, mayor será la actitud hacia estas fuentes de energía.

La H5 queda confirmada, estableciéndose una relación fuerte entre las variables consideradas, con un coeficiente estandarizado significativo y con un valor superior a 0,5 ( $8=$ 0,571; sign. =0,00). Así se descubre que cuanto más favorable es la actitud hacia las energías renovables mayor es la intención de compra hacia algunas de las instalaciones consideradas. 
Los resultados obtenidos por el modelo propuesto son acordes con los estudios anteriores realizados y mencionados en el epígrafe de revisión de la literatura científica.

\section{Conclusiones y recomendaciones finales}

La presente investigación ha modelizado el comportamiento del consumidor ante las energías renovables, encontrando apoyo en la teoría de la acción razonada (TRA), al relacionar de manera positiva el conocimiento previo sobre las energías renovables con la actitud. Además, se tomó como variable exógena el comportamiento pro-ambiental, demostrando una relación fuerte entre esta variable y el conocimiento sobre las energías renovables. Los resultados alcanzados son consistentes con otros resultados de la investigación sobre medio ambiente y la comercialización, tales como los de Egea y Frutos (2013), Pagiaslis y Krontalis (2014) o Lin y Syrgabayeva (2016). La principal diferencia con estos estudios es que ellos predicen la disposición a pagar más por el consumo de energías renovables, mientras que nuestro estudio analiza la intención de compra demostrando que entre la preocupación por el medio, la actitud y la intención, no se da la misma fuerza en la relación, ya que la instalación de energías renovables supone un esfuerzo, que frena tanto la actitud como la intención de compra del consumidor. Parece que, aunque los consumidores que se preocupan por el medio ambiente y tienen conocimiento sobre las energías renovables se ven influidos por variables situacionales, que disminuyen tanto la actitud hacia las mismas como la intención de compra de estas.

Uno de los problemas que pueden encontrar las empresas comercializadoras es la inversión inicial que frena la compra de estas instalaciones.

Otro problema es la falta de credibilidad y de capacidad para convencer a los consumidores de las ventajas de las energías renovables. El conocimiento de estas ventajas generará actitudes más favorables y más estables, que estimularán a su vez el comportamiento de compra de tales sistemas energéticos.

Ya que las actitudes parten del conocimiento, estas empresas deben utilizar anuncios que informen y convenzan sobre el ahorro energético y económico que a medio y largo plazo supone su instalación en el hogar. Una forma de conseguir esto sería ofrecer en las campañas una comparación entre sistemas convencionales y los basados en energías "verdes", añadiendo el equivalente monetario que supone el ahorro generado durante un año.

\section{Limitaciones y futuras líneas de investigación}

La legislación y el funcionamiento de las empresas eléctricas en otros países, hace que las investigaciones tomen en consideración situaciones que en España no ocurren (como la opción de consumir energías renovables mientras se pague más en la factura). No obstante, se creyó que se trata de un tema interesante y controvertido que debe ser analizado con mayor profundidad.

Sería importante poner a prueba la solidez del modelo, ya que el tamaño muestral utilizado por su facilidad de acceso no nos permite inferir los resultados obtenidos a otras provincias españolas. De esta manera la investigación mejoraría la validez externa de los resultados alcanzados.

Por último, se propone analizar la efectividad de los anuncios publicitarios de empresas y administraciones públicas centradas en energías "verdes" mediante la metodología de 
eyetracking y otras medidas psicofisiológicas (resonancias magnéticas funcionales, electroencefalogramas, respuesta galvánica de la piel,...).

\section{Referências}

Ajzen, I. (1991). The theory of planned behavior. Organizational Behavior and Human Decision Processes, 50, 179-211. doi: 10.1016/0749-5978(91)90020-T

Ajzen, I., \& Fishbein, M. (1980). Understanding attitudes and predicting social behaviour. New Jersey: Prentice Hall.

Alonso, J. A. (2013). El planeta Tierra en peligro (calentamiento global, cambio climático, soluciones). Alicante: Editorial Club Universitario.

Bamberg, S. (2003). How does environmental concern influence specific environmentally related behaviors? A new answer to an old question. Journal of Environmental Psychology, 23(1), 21-32. doi: 10.1016/S0272-4944(02)00078-6

Blake, J. (1999). Overcoming the "value-action gap" in environmental policy: Tensions between national policy and local experience. Local Environment. The International Journal of Justice and Sustainability, 4(3), 257-278. doi: 10.1080/13549839908725599

Bruner, G. C. (2009). Marketing scales handbook. A compilation of multi-ltem measures for consumer behaviour \& advertising research. (Vol. 5). Carbondale, IL: GCBII Productions.

Carrero, I., Valor, C. \& Redondo, R. (2015). Los determinantes de la compra de productos con etiquetas de contenido social y ambiental. CIRIEC-Espana, 83, 235-250.

Camarena Urbano, F. M. M. (2013). Ideología política y valores: Su relación con las creencias y conductas hacia el ambiente (Tese de doutoramento não publicada). Pontificia Universidad Católica del Perú, Perú.

Castañeda, J. S. (2014). Contextualización y enfoques en el estudio de comportamientos proambientales o ecológicos con miras a la perfilación del consumidor verde. Suma de Negocios, 5(10), 34-39. doi:10.1016/S2215-910X(14)70007-2

Chamorro, A., Rubio, S. \& Miranda, F. J. (2009). Characteristics of research on green marketing. Business Strategy and the Environment, 18(4), 223-239. doi: 10.1002/bse.571

Chan, D. C., Wu, A. M. \& Hung, E. P. W. (2010). Invulnerability and the intention to drink and drive: An application of the theory of planned behavior. Accident Analysis \& Prevention, 42(6), 1549-1555. doi: 10.1016/j.aap.2010.03.011

Churchill Jr, G. A. (1979). A paradigm for developing better measures of marketing constructs. Journal of Marketing Research, 16(1), 64-73.

Claudy, M. C., Peterson, M., \& O'Driscoll, A. (2013). Understanding the attitude-behavior gap for renewable energy systems using behavioral reasoning theory. Journal of Macromarketing, 33(4), 273-287.

Cortés-Peña, O. F. (2016). Comportamiento proambiental y pensamiento económico en la construcción del desarrollo sostenible. Cultura Educación y Sociedad, 2(1).

Clark, C.F., Kotchen, M.J. \& Moore, M.R. (2003). Internal and external influences on pro-environmental behavior: Participation in a green electricity program. Journal of Environmental Psychology, 23, 237-246. doi: 10.1016/S0272-4944(02)00105-6

Diamantopoulos, A., Schlegelmilch, B. B., Sinkovics R. R. \& Bohlen, G. M. (2003). Can sociodemographics still play a role in profiling green consumers? A review of the evidence and an empirical investigation. Journal of Business Research, 56(6), 465-480. doi: 10.1016/S01482963(01)00241-7

Dono, J., Webb, J. \& Richardson, B. (2010). The relationship between environmental activism, proenvironmental behaviour and social identity. Journal of Environmental Psychology, 30(2), 178180. doi: 10.1016/j.jenvp.2009.11.006

Dunlap, R.E. \& Van Liere, K. (1978). The new environmental paradigm: A proposed measuring instrument and preliminary results. The Journal of Environmental Education, 9(4), 10-19. doi: 10.1080/00958964.1978.10801875

Egea, J. M. O. \& Frutos, N. G. (2013). Toward consumption reduction: An environmentally motivated perspective. Psychology \& Marketing, 30(8), 660-675. doi: 10.1002/mar.20636/epdf

Fielding, K. S., Terry, D. J., Masser, B. M. \& Hogg, M. A. (2008). Integrating social identity theory and the theory of planned behaviour to explain decisions to engage in sustainable agricultural practices. British Journal of Social Psychology, 47(1), 23-48. doi: 10.1348/014466607X206792/full 
Frederiks, E. R., Stenner, K. \& Hobman, E. V. (2015). Household energy use: Applying behavioural economics to understand consumer decision-making and behaviour. Renewable and Sustainable Energy Reviews, 41, 1385-1394. doi: 10.1016/j.rser.2014.09.026

García, I. (2012). Estudio cualitativo de la adopción de la biomasa por el consumidor final. La perspectiva de los productores y distribuidores (Trabajo de investigación tutelada). Universidad de Granada, España.

García, L., Orellana, O., Miljánovich, M., Yanac, E., Herrera, E., Espinoza, M., ... \& Fernandini, P. (2016). Compromiso y comportamiento ecológico en estudiantes universitarios de Lima y Huaraz. Revista de Investigación en Psicología, 18(2), 57-70.

Gardner, G. T. \& Stern, P. C. (2008). The short list: The most effective actions us households can take to curb climate change. Environment: Science and Policy for Sustainable Development, 50(5), 12-25. doi: $10.3200 /$ ENVT.50.5.12-25

Gould, L. C., Gardner, G. T., Deluca, D. R., Tiemann, A. R., Doob, L. W. \& Stolwijk, J. A. (1988). Perceptions of technological risks and benefits. New York: Russell Sage Foundation.

Guagnano G. A., Stern P. C. \& Dietz T. (1995). Influences on attitude-behavior relationships: A natural experiment with curbside recycling. Environment and Behavior, 27(5), 699-718. doi: 10.1177/0013916595275005

Ha, H. Y. \& Janda, S. (2012). Predicting consumer intentions to purchase energy-efficient products. Journal of Consumer Marketing, 29(7), 461-469. doi: 10.1108/07363761211274974

Hansla, A. Gamble, A. Juliusson, A. \& Gärling, T. (2008). The relationships between awareness of consequences, environmental concern, and value orientations. Journal of Environmental Psychology, 28(1), 1-9. doi: 10.1016/j.jenvp.2007.08.004

Hines, J.M., Hungerford, H.R. \& Tomera, A.N. (1987). Analysis and synthesis of research on responsible environmental behavior: A meta-analysis. The Journal of Environmental Education, 18(2), 1-8. doi: 10.1080/00958964.1987.9943482

Huang, L., Gursoy, D. \& Honggang, X. (2014). Impact of personality traits and involvement on prior knowledge. Annals of Tourism Research, 48, 42-57. doi: 10.1016/j.annals.2014.05.010

Imhoff, D., Gariglio, C., Ponce, V., Díaz, B. \& Pilatti, A. (2014). Environmental activism: Relationship with psycho-social and psycho-political variables among activists and non-activists from Argentina/Activismo ambiental: Relación con variables psicosociales y psicopolíticas en activistas y no activistas de Argentina. Psyecology, 5 (2-3), 350-374. doi: 10.1080/21711976.2014.957541

Izagirre-Olaizola, J., Fernández-Sainz, A. \& Vicente-Molina, M. A. (2013). Antecedentes y barreras a la compra de productos ecológicos. Universia Business Review, 38, 108-127.

Jamieson, F.M. \& Bass, F.M. (1989). Adjusting stated intention measures to predict trial purchase of new products: A comparison of models and methods. Journal of Marketing Research, 26(3), 336345.

Jones, R. E., \& Dunlap, R. E. (1992). The social bases of environmental concern: Have they changed over time? Rural sociology, 57(1), 28-47. doi: 10.1111/j.1549-0831.1992.tboo455.x

Karp, D. (1996). Values and their effect on pro-environmental behavior. Environment and Behavior, 28(1), 111-133. doi: 10.1177/0013916596281006

Kelly, S., Mcdonald, R. \& Winnifred, R. L. (2008). Theory of planned behaviour, identity and intentions to engage in environmental activism. Journal of Environmental Psychology, 28(4), 318-326. doi: 10.1016/j.jenvp.2008.03.003

Li, H., Jenkins-Smith, H. C., Silva, C. L., Berrens, R. P. \& Herron, K. G. (2009). Public support for reducing US reliance on fossil fuels: Investigating household willingness-to-pay for energy research and development. Ecological Economics, 68(3), 731-742. doi: 10.1016/j.ecolecon.2008.06.005

Lin, C. Y. \& Syrgabayeva, D. (2016). Mechanism of environmental concern on intention to pay more for renewable energy: Application to a developing country. Asia Pacific Management Review, 21(3), 125-134.

Maloney, M.P. \& Ward, M.P. (1973). Ecology: Let's hear from the people: An objective scale for the measurement of ecological attitudes and knowledge. American Psychologist, 28(7), 583-586. doi: 10.1037/hoo34936

Miguens, M. J. L., González, P. Á. \& Vázquez, E. G. (2015). Conocimiento, valores e intenciones como determinantes del comportamiento ecológico. Revista Internacional de Sociología, 73(3), 018. doi: $10.3989 /$ ris.2015.73.3.e018

Mitra, S. (2008). Patent and food security - opening the Pandora's box. Journal of Intellectual Property Rights, 13, 145-151. 
Moreno, B. \& López, A. (2008). Las energías renovables: Perspectivas e impacto sobre el empleo en Asturias. Revista de Estudios Regionales, 83, 177-195.

Nelson, A., Bishop, M. \& Gruen, T. (2014). Who pays more (or less) for pro-environmental consumer goods? Using the auction method to assess actual willingness-to-pay. Journal of Environmental Psychology, 40, 218-227. doi: 10.1016/j.jenvp.2014.06.010

Oreskes, N. (2004). The scientific consensus on climate change. Science, 306 (5702), 16-86. doi: $10.1126 /$ science. 1103618

Pelletier, L.G., Tuson, K.M., Green-Demers, I., Noels, K, \& Beaton, A.M (1998). Why are you doing things for the environment? The motivation toward the environment scale (MTES). Journal of Applied Social Psychology, 28(5), 437-468. doi: 10.1111/j.1559-1816.1998.tbo1714.x

Pagiaslis, A. \& Krontalis, A. K. (2014). Green consumption behavior antecedents: Environmental concern, knowledge, and beliefs. Psychology \& Marketing, 31(5), 335-348. doi: 10.1002/mar.20698

Podsakoff, P. M., MacKenzie, S. B., Lee, J. Y. \& Podsakoff, N. P. (2003). Common method biases in behavioral research: A critical review of the literature and recommended remedies. Journal of Applied Psychology, 88(5), 879-903. doi: 10.1037/0021-9010.88.5.879

Ranganathan, C. \& Ganapathy, S. (2002). Key dimensions of business-to-consumer web sites. Information and Management, 39 (6), 457-465. doi: 10.1016/S0378-7206(01)00112-4

Rifon, N.J., LaRose, R. \& Choi, S.M. (2005). Your privacy is sealed: Effects of web privacy seal on trust and personal disclosures. The Journal of Consumer Affairs, 39(2), 339-362. doi: 10.1111/j.17456606.2005.00018.x

Rijnsoever, F. \& Farla, J. (2014). Identifying and explaining public preferences for the attributes of energy technologies. Renewable and Sustainable Energy, 31, 71-82. doi: 10.1016/j.rser.2013.11.048

Sardianou, E. \& Genoudi, P. (2013). Which factors affect the willingness of consumers to adopt renewable energies?. Renewable Energy, 57, 1-4. doi: 10.1016/j.renene.2013.01.031

Séguin, C., Pelletier, L. G. \& Hunsley, J. (1999). Predicting environmental behaviors: The influence of self-determination and information about perceived environmental health risk. Journal of Applied Social Psychology, 29(8), 1582-1604. doi: 10.1111/j.1559-1816.1999.tb02043.x

Senbel, M., Douglas, V. \& Blair, E. (2014). Social mobilization of climate change: University students conserving energy through multiple pathways for peer engagement. Journal of Environmental Psychology, 38, 84-93. doi: 10.1016/j.jenvp.2014.01.001

Sengers, F., Raven, R.P. \& Van, A. (2010). From riches to rags: Biofuels, media discourses, and resistance to sustainable energy technologies. Energy Policy, 38(9), 5013-5027. doi: 10.1016/j.enpol.2010.04.030

Slovic, P. (1987). Perception of Risk. Science, 236 (4799), 280-285.

Stern, P., Dietz, T., Gregory A. \& Guagnano, C. (1995). The new ecological paradigm in socialpsychological context. Environment and Behavior, 27 (6), 723-743. doi: 10.1177/0013916595276001

Stigka, E. K., Paravantis, J. A., \& Mihalakakou, G. K. (2014). Social acceptance of renewable energy sources: A review of contingent valuation applications. Renewable and Sustainable Energy Reviews, 32, 100-106. doi: 10.1016/j.rser.2013.12.026

Venkatesh, V., Morris, M. G., Davis, G. B., \& Davis, F. D. (2003). User acceptance of information technology: Toward a unified view. MIS Quarterly, 27(3), 425-478.

Vringer, K., Aalbers, T. \& Blok, K. (2007). Household energy requirement and value patterns. Energy Policy, 35(1), 553-566. doi: 10.1016/j.enpol.2005.12.025

Whitmarsh, L. \& O'Neill, S. (2010). Green identity, green living? The role of pro-environmental selfidentity in determining consistency across diverse pro-environmental behaviours. Journal of Environmental Psychology, 30(3), 305-314. doi: 10.1016/j.jenvp.2010.01.003

Wolske, K. S., Stern, P. C. \& Dietz, T. (2017). Explaining interest in adopting residential solar photovoltaic systems in the United States: Toward an integration of behavioral theories. Energy Research \& Social Science, 25, 134-151. doi: 10.1016/j.erss.2016.12.023

Zaichkowsky, J. L. (1985). Measuring the involvement construct. Journal of Consumer Research, 12(3), 341-352. doi: 10.1086/208520

Zsóka, Á., Szerényi, Z. M., Széchy, A. \& Kocsis, T. (2013). Greening due to environmental education? Environmental knowledge, attitudes, consumer behavior and everyday pro-environmental activities of Hungarian high school and university students. Journal of Cleaner Production, 48, 126-138. doi: 10.1016/j.jclepro.2012.11.030 
Este trabajo recibió financiación del proyecto de Excelencia de la Junta de Andalucía "El neuromarketing como herramienta de comprensión de los mecanismos cognitivos y afectivos que rigen el procesamiento de la comunicación tendente a conseguir un comportamiento de consumo medioambientalmente responsable" (P12-SEJ-1980).

DIEGO GÓMEZ CARMONA is a doctoral student in Marketing and Market Research and holds a P.D.I in Business Sciences from the University of Granada (Spain). Although his main research interest is ecological consumer behavior and environmental communication. His recent works have appeared in national conferences (CIMCYC). Dirección institucional: Marketing and Market Research Department, Faculty of Economics and Business Administration, University of Granada, University campus. La Cartuja, s/n, E-18071 Granada, Spain.

FRANCISCO MUÑOZ-LEIVA is lecturer in Marketing and Market Research and holds a Ph.D. in Business Sciences from the University of Granada (Spain). Although his main research interest is Internet consumer behaviour and Internet acceptance, he has also published papers on other topics. His recent works have appeared in journals such as International Journal of Advertising, Industrial Management \& Data System, Soft Computing, Current Issues in Tourism, Expert Systems with Applications, Online Information Review, Information \& Management, Computers in Human Behavior, International Journal of Information Management, The Service Industries Journal, Quality \& Quantity, among others. Dirección institucional: Marketing and Market Research Department, Faculty of Economics and Business Administration, University of Granada, University campus. La Cartuja, s/n, E-18071 Granada, Spain.

FRANCISCO LIEBANA-CABANILLAS is an Assistant Professor in the Department of Marketing and Market Research at the University of Granada (Spain) since 2000 and holds a Ph.D. in Business Sciences at this university. The results of which are reflected in various papers including Expert Systems With Applications, Service Industries Journal, Industrial Management \& Data Systems, International Journal of Information Management, Technology Analysis \& Strategic Management, Computers in Human Behavior, The Service Industries Journal, Information Systems and e-Business Management, among others; as well as in national and international conferences. Dirección institucional: Marketing and Market Research Department, Faculty of Economics and Business Administration, University of Granada, University campus. La Cartuja, s/n, E-18071 Granada, Spain.

Submetido em 16 novembro 2016

Aceite em 27 julho 2017 
ANEXO 1. Cuestionario

\begin{tabular}{|c|c|c|}
\hline $\begin{array}{l}\text { Por favor, puntúe la frecuencia con la que participa en las siguientes } \\
\text { actividades, donde } 1 \text { es nunca y } 5 \text { es siempre }\end{array}$ & Nunca & Siempre \\
\hline Participo en actividades que cuidan del medio ambiente & \multicolumn{2}{|c|}{ (1) (2) (3) (4) (5) } \\
\hline $\begin{array}{l}\text { Participo en manifestaciones públicas para defender el medio } \\
\text { ambiente }\end{array}$ & \multicolumn{2}{|c|}{ (1) (2) (3) (4) (5) } \\
\hline Hago trabajo voluntario para un grupo ambiental & \multicolumn{2}{|c|}{ (1) (2) (3) (4) (5) } \\
\hline Movilizo a las personas para la conservación de los espacios públicos & \multicolumn{2}{|c|}{ (1) (2) (3) (4) (5) } \\
\hline Hablo sobre la importancia del medio ambiente con las personas & \multicolumn{2}{|c|}{ (1) (2) (3) (4) (5) } \\
\hline $\begin{array}{l}\text { Evito usar productos fabricados por una empresa cuando sé que esa } \\
\text { empresa está contaminando el medio ambiente }\end{array}$ & \multicolumn{2}{|c|}{ (1) (2) (3) (4) (5) } \\
\hline $\begin{array}{l}\text { Cuando estoy en casa, dejo las lámparas encendidas en lugares que } \\
\text { no son necesarias }\end{array}$ & \multicolumn{2}{|c|}{ (1) (2) (3) (4) (5) } \\
\hline Evito desperdiciar energía & \multicolumn{2}{|c|}{ (1) (2) (3) (4) (5) } \\
\hline Dejo la televisión encendida incluso cuando nadie la está viendo & \multicolumn{2}{|c|}{ (1) (2) (3) (4) (5) } \\
\hline Apago la lámpara cuando salgo de una habitación & \multicolumn{2}{|c|}{ (1) (2) (3) (4) (5) } \\
\hline $\begin{array}{l}\text { Cuando abro la nevera, evito quedarme con la puerta abierta mucho } \\
\text { tiempo para no gastar energía }\end{array}$ & \multicolumn{2}{|c|}{ (1) (2) (3) (4) (5) } \\
\hline Evito desperdiciar los recursos naturales & \multicolumn{2}{|c|}{ (1) (2) (3) (4) (5) } \\
\hline $\begin{array}{l}\text { Cuando tengo ganas de comer alguna cosa que no sé lo que es, abro } \\
\text { la nevera y me quedo mirando lo que hay }\end{array}$ & \multicolumn{2}{|c|}{ (1) (2) (3) (4) (5) } \\
\hline $\begin{array}{l}\text { Evito encender varios aparatos eléctricos al mismo tiempo en los } \\
\text { horarios de mayor consumo de energía }\end{array}$ & \multicolumn{2}{|c|}{ (1) (2) (3) (4) (5) } \\
\hline $\begin{array}{l}\text { Puntúe su grado de acuerdo o desacuerdo con las siguientes } \\
\text { afirmaciones, donde } 1 \text { es totalmente en desacuerdo y } 5 \text { totalmente } \\
\text { de acuerdo }\end{array}$ & $\begin{array}{c}\text { Totalmente } \\
\text { en desacuerdo }\end{array}$ & $\begin{array}{l}\text { Totalmente } \\
\text { de acuerdo }\end{array}$ \\
\hline Conozco bastante sobre diversas tecnologías energéticas & \multicolumn{2}{|c|}{ (1) (2) (3) (4) (5) } \\
\hline No considero que conozca las diferentes tecnologías energéticas & \multicolumn{2}{|c|}{ (1) (2) (3) (4) (5) } \\
\hline $\begin{array}{l}\text { Entre mi círculo de amigos yo soy uno de los que más suelen saber } \\
\text { sobre tecnologías energéticas }\end{array}$ & \multicolumn{2}{|c|}{ (1) (2) (3) (4) (5) } \\
\hline $\begin{array}{l}\text { En comparación con la mayoría de las otras personas, sé menos sobre } \\
\text { diversas tecnologías energéticas }\end{array}$ & \multicolumn{2}{|c|}{ (1) (2) (3) (4) (5) } \\
\hline $\begin{array}{l}\text { Cuando se trata de tecnologías yo realmente no conozco mucho } \\
\text { acerca de ellas }\end{array}$ & \multicolumn{2}{|c|}{ (1) (2) (3) (4) (5) } \\
\hline $\begin{array}{l}\text { Puntúe su grado de acuerdo o desacuerdo con las siguientes } \\
\text { afirmaciones, donde } 1 \text { es totalmente en desacuerdo y } 5 \text { totalmente } \\
\text { de acuerdo. }\end{array}$ & $\begin{array}{l}\text { Totalmente } \\
\text { en desacuerdo }\end{array}$ & $\begin{array}{l}\text { Totalmente } \\
\text { de } \\
\text { acuerdo }\end{array}$ \\
\hline $\begin{array}{l}\text { Las energías renovables pueden generar beneficios económicos } \\
\text { indirectos, como por ejemplo el derivado del ahorro energético }\end{array}$ & \multicolumn{2}{|c|}{ (1) (2) (3) (4) (5) } \\
\hline Con las energías renovables se puede abastecer a todo el mundo & \multicolumn{2}{|c|}{ (1) (2) (3) (4) (5) } \\
\hline Las energías renovables disponen de la tecnología necesaria & \multicolumn{2}{|c|}{ (1) (2) (3) (4) (5) } \\
\hline $\begin{array}{l}\text { Las energías renovables no son sensibles a cambios en el precio del } \\
\text { combustible }\end{array}$ & \multicolumn{2}{|c|}{ (1) (2) (3) (4) (5) } \\
\hline Las renovables generan empleo & \multicolumn{2}{|c|}{ (1) (2) (3) (4) (5) } \\
\hline Las energías renovables son fuentes fiables de energía & \multicolumn{2}{|c|}{ (1) (2) (3) (4) (5) } \\
\hline $\begin{array}{l}\text { Con las energías renovables se garantiza el suministro constante de } \\
\text { energía }\end{array}$ & \multicolumn{2}{|c|}{ (1) (2) (3) (4) (5) } \\
\hline Las energías renovables tienen una larga vida útil en la propia casa. & \multicolumn{2}{|c|}{ (1) (2) (3) (4) (5) } \\
\hline Tienen un corto periodo de amortización. & \multicolumn{2}{|c|}{ (1) (2) (3) (4) (5) } \\
\hline $\begin{array}{l}\text { Supone una gran inversión obtener algún tipo de energía renovable } \\
\text { para nuestra casa }\end{array}$ & \multicolumn{2}{|c|}{ (1) (2) (3) (4) (5) } \\
\hline Las energías renovables tienen mucho coste de mantenimiento en la & \multicolumn{2}{|c|}{ (1) (2) (3) (4) (5) } \\
\hline
\end{tabular}




\begin{tabular}{|l|c|c|}
\hline propia casa & \multicolumn{2}{|c|}{ (1) (2) (3) (4) (5) } \\
\hline $\begin{array}{l}\text { Son necesarios algunos ajustes en la propia casa para dar cabida a } \\
\text { una tecnología }\end{array}$ & \multicolumn{2}{|c|}{ (1) (2) (3) (4) (5) } \\
\hline Las energías renovables necesitan mucho espacio & \multicolumn{2}{|c|}{ (1) (2) (3) (4) (5) } \\
\hline Las renovables provocan mucho ruido & \multicolumn{2}{|c|}{ (1) (2) (3) (4) (5) } \\
\hline Las renovables causan efecto negativo sobre el paisaje (4) \\
\hline Las energías renovables tienen un alto precio & $\begin{array}{l}\text { Muy } \\
\text { positiva }\end{array}$ \\
\hline $\begin{array}{l}\text { Valore su actitud hacia los sistemas de ahorro basados en energías } \\
\text { renovables. (donde } 1 \text { es muy negativa y } 5 \text { es muy positiva) }\end{array}$ & $\begin{array}{l}\text { Muy } \\
\text { negativa }\end{array}$ & \multicolumn{1}{|c|}{ (1) (2) (3) (4) (5) } \\
\hline $\begin{array}{l}\text { Mi actitud hacia el uso de sistemas de ahorro basados en energías } \\
\text { renovables es ... }\end{array}$ & $\begin{array}{l}\text { Totalmente } \\
\text { en } \\
\text { desacuerdo }\end{array}$ & $\begin{array}{l}\text { Totalmente } \\
\text { de acuerdo }\end{array}$ \\
\hline $\begin{array}{l}\text { Valore su grado de acuerdo con las siguientes afirmaciones. (donde } \\
\text { es totalmente en desacuerdo y } 5 \text { es totalmente de acuerdo) }\end{array}$ & (1) (2) (3) (4) (5) \\
\hline $\begin{array}{l}\text { En un futuro próximo, estaría dispuesto a comprar un sistema de } \\
\text { ahorro energético basado en energías renovables }\end{array}$ & (1) (2) (3) (4) (5) \\
\hline $\begin{array}{l}\text { Cuando tenga que decidir por un nuevo sistema de calefacción para } \\
\text { mi casa, mi intención es comprar uno que funcione con biomasa }\end{array}$ & (1) (2) (3) (4) (5) \\
\hline $\begin{array}{l}\text { Cuando tenga que decidir por un nuevo sistema para el agua caliente } \\
\text { sanitaria de mi casa, mi intención es comprar placas solares. }\end{array}$ & (1) (2) (3) (4) (5) \\
\hline $\begin{array}{l}\text { Cuando tenga que decidir por un sistema de ahorro de electricidad } \\
\text { para mi casa, mi intención es comprar placas fotovoltaicas. }\end{array}$ &
\end{tabular}

\section{ANEXO 2. Coeficientes del modelo}

\begin{tabular}{|lll|c|c|c|}
\hline & Coeficientes & Sin & Estandarizados (B) & Sign. \\
\hline $\begin{array}{l}\text { Conocimiento_P } \\
\text { revio }\end{array}$ & $<---$ & $\begin{array}{l}\text { Comportamiento_pro- } \\
\text { ambiental }\end{array}$ & 0,769 & 0,725 & 0,000 \\
\hline Actitud & $<---$ & Conocimiento_previo & 0,366 & 0,312 & 0,008 \\
\hline Actitud & $<--$ & $\begin{array}{l}\text { Comportamiento_pro- } \\
\text { ambiental }\end{array}$ & 0,393 & 0,316 & 0,016 \\
\hline $\begin{array}{l}\text { Intención_de_co } \\
\text { mpra }\end{array}$ & $<---$ & Actitud & 0,419 & 0,571 & 0,000 \\
\hline $\begin{array}{l}\text { Intención_de_co } \\
\text { mpra }\end{array}$ & $<---$ & $\begin{array}{l}\text { Comportamiento_pro- } \\
\text { ambiental }\end{array}$ & 0,288 & 0,315 & 0,000 \\
\hline Act1 & $<--$ & Actitud & 1,000 & 1,000 & \\
\hline Int1 & $<---$ & Intención_de_compra & 1,000 & 0,729 & \\
\hline Int2 & $<--$ & Intención_de_compra & 1,006 & 0,727 & 0,000 \\
\hline Int3 & $<---$ & Intención_de_compra & 1,027 & 0,740 & 0,000 \\
\hline Int4 & $<--$ & Intención_de_compra & 0,909 & 0,628 & 0,000 \\
\hline CP1 & $<---$ & Conocimiento_previo & 1,000 & 0,796 & 0,000 \\
\hline CP2 & $<--$ & Conocimiento_previo & 0,953 & 0,755 & 0,000 \\
\hline CP3 & $<---$ & Conocimiento_previo & 0,971 & 0,773 & 0,000 \\
\hline CP4 & $<--$ & Conocimiento_previo & 1,015 & 0,790 & 0,000 \\
\hline CP5 & $<---$ & Conocimiento_previo & 0,976 & 0,784 & \\
\hline ahorro & $<---$ & $\begin{array}{l}\text { Comportamiento_pro- } \\
\text { ambiental }\end{array}$ & 1,000 & 0,776 & 0,000 \\
\hline activismo & $<---$ & $\begin{array}{l}\text { Comportamiento_pro- } \\
\text { ambiental }\end{array}$ & 0,809 & 0,624 & \\
\hline
\end{tabular}

